The Astrophysical Journal, 672:1202-1208, 2008 January 10

(C) 2008. The American Astronomical Society. All rights reserved. Printed in U.S.A.

\title{
DETECTION OF A THIRD PLANET IN THE HD 74156 SYSTEM USING THE HOBBY-EBERLY TELESCOPE ${ }^{1}$
}

\author{
Jacob L. Bean, ${ }^{2,3}$ Barbara E. McArthur, ${ }^{2}$ G. Fritz Benedict, ${ }^{2}$ and Amber Armstrong ${ }^{2}$ \\ Received 2007 August 4; accepted 2007 September 11
}

\begin{abstract}
We report the discovery of a third planetary-mass companion to the G0 star HD 74156. High-precision radial velocity measurements made with the Hobby-Eberly Telescope aided the detection of this object. The best-fit triple-Keplerian model to all the available velocity data yields an orbital period of 347 days and a minimum mass of $0.4 M_{\text {Jup }}$ for the new planet. We determine revised orbital periods of 51.7 and 2477 days and minimum masses of 1.9 and $8.0 M_{\text {Jup }}$, respectively, for the previously known planets. Preliminary calculations indicate that the derived orbits are stable, although all three planets have significant orbital eccentricities $(e=0.64,0.43$, and 0.25$)$. With our detection, HD 74156 becomes the eighth normal star known to host three or more planets. Further study of this system's dynamical characteristics will likely give important insight into planet formation and evolutionary processes.
\end{abstract}

Subject headings: planetary systems — stars: individual (HD 74156) — techniques: radial velocities

\section{INTRODUCTION}

More than 200 planets have been discovered around nearby stars other than the Sun using the radial velocity method. ${ }^{4}$ Despite the high number of known exoplanets, the hunt for additional planets around nearby stars with the radial velocity method remains a fundamental research area. Planet candidates around nearby stars are the most suitable for follow-up study such as photometric monitoring for transits (e.g., Henry et al. 2000; Charboneau et al. 2000; Castellano 2000; Bouchy et al. 2005; Sato et al. 2005; Shankland et al. 2006; Lòpez-Morales et al. 2006; Gillon et al. 2007), astrometric perturbation measurements (e.g., Benedict et al. 2002, 2006; McArthur et al. 2004; Bean et al. 2007), thermal emission searches (e.g., Deming et al. 2005; Harrington et al. 2006; Richardson et al. 2007; Knutson et al. 2007; Cowan et al. 2007), attempted direct imaging detection (e.g., Janson et al. 2007), and dynamical characterization (e.g., Laughlin \& Adams 1999; Chiang \& Murray 2002; Adams \& Laughlin 2006; Ford et al. 2005; Barnes \& Greenberg 2006). The results of these types of investigations improve our understanding of planet formation and evolution.

Radial velocity planet searches are also a crucial component of the quest to determine the uniqueness of our own solar system. It should be noted that only recently have some surveys achieved the necessary precision and timespan to detect true Jupiter analogs (i.e., planets with roughly the same mass and orbital semimajor axis), and it is not yet possible to detect analogs of any of the other planets in our solar system. Therefore, the continuation and improvement of radial velocity planet searches should be a top priority.

After our successful detection of an additional companion to $\rho^{1} \mathrm{Cnc}$ (= $55 \mathrm{Cnc}$; McArthur et al. 2004), we began a small radial velocity monitoring program in 2004 with the goal of finding additional planets in already known planetary systems. Our meth-

\footnotetext{
${ }^{1}$ Based on data obtained with the Hobby-Eberly Telescope (HET). The HET is a joint project of the University of Texas at Austin, the Pennsylvania State University, Stanford University, Ludwig-Maximilians-Universität Muenchen, and Georg-August-Universität Göttingen. The HET is named in honor of its principal benefactors, William P. Hobby and Robert E. Eberly.

2 Department of Astronomy and McDonald Observatory, University of Texas, 1 University Station, C1402, Austin, TX 78712.

${ }^{3}$ Now at the Institut für Astrophysik, Georg-August-Universität Göttingen, Friedrich-Hund-Platz 1, 37077 Göttingen, Germany; bean@astro.physik.unigoettingen.de.

${ }^{4}$ A regularly updated list of reported extrasolar planets is maintained at the Extrasolar Planets Encyclopedia Web site, http://exoplanet.eu.
}

odology was to take advantage of the Hobby-Eberly Telescope's (HET) queue-scheduled operations to obtain high-cadence radial velocities of selected systems. In addition, we chose to obtain multiple radial velocity measurements per observational epoch to reduce the impact of both statistical and stellar noise on the search for previously hidden planets. This intensive technique limits the number of possible targets, but is a natural complement to the larger planet search programs.

In this paper we report the first result from this project, the detection of a third planet around HD 74156. In $\S 2$ we give the properties and background information on the star. In $\S 3$ we describe our radial velocity measurements with the HET. We present our analysis that yields the detection of the new planet in $\S 4$. We conclude with a discussion of our result in $\S 5$.

\section{HD 74156 PROPERTIES}

According to the Hipparcos catalog (ESA 1997), HD 74156 (HIP 42723) is a G0 star about $65 \mathrm{pc}$ from the Sun $\left(\pi_{\mathrm{abs}}=\right.$ $15.49 \pm 1.10$ mas) with visual magnitude $V=7.61$ and color $B-V=0.59$. Naef et al. (2004) previously reported the discovery of two planetary-mass companions to the star based on its radial velocity variations. The planets had estimated minimum masses $M_{b} \sin i=1.9 M_{\text {Jup }}$ and $M_{c} \sin i=6.2 M_{\text {Jup }}$ assuming the primary's mass $M_{A}=1.27 M_{\odot}$. The lower mass planet is in a short-period orbit $\left(P_{b}=51.6\right.$ days $)$, while the higher mass planet has a longer orbital period $\left(P_{c}=2025\right.$ days). Both planets' orbits were reported as highly eccentric $(e=0.64$ and 0.58$)$.

Valenti \& Fischer (2005) determined an iron abundance $[\mathrm{Fe} / \mathrm{H}]=$ +0.13 for HD 74156. This result agrees with that from Santos et al. (2003), who found $[\mathrm{Fe} / \mathrm{H}]=+0.15$. Therefore, it is metalrich compared to the solar neighborhood, but average among host stars to high-mass planets (Fischer \& Valenti 2005).

Naef et al. (2004) suggested that HD 74156 might be slightly evolved based on its large estimated luminosity relative to its spectral type. Takeda et al. (2007) report an age of $3.7 \pm 0.4 \mathrm{Gyr}$ based on a comparison of the star's properties with theoretical models. This age estimate implies that HD 74156 is still on the main sequence, although ages for field stars are notoriously difficult to determine. Naef et al. (2004) reported seeing no emission in the $\mathrm{Ca}$ II $\mathrm{H}$ line core, which is consistent with the star having at least an intermediate age.

Takeda et al. (2007) also proposed a mass $M_{A}=1.24 \pm$ $0.04 M_{\odot}$ for HD 74156. This is consistent with the mass adopted 
TABLE 1

HET Radial Velocities for HD 74156

\begin{tabular}{|c|c|}
\hline HJD $-2,450,000.0$ & $\begin{array}{c}\mathrm{RV} \\
\left(\mathrm{m} \mathrm{s}^{-1}\right)\end{array}$ \\
\hline 3342.8969 & $-47.28 \pm 3.84$ \\
\hline 3347.0019. & $-41.26 \pm 2.58$ \\
\hline $3355.8330 \ldots$ & $-34.26 \pm 2.89$ \\
\hline $3357.8444 \ldots$ & $-35.63 \pm 2.91$ \\
\hline $3359.8504 \ldots$ & $-28.42 \pm 4.95$ \\
\hline $3360.9748 \ldots$ & $-37.04 \pm 3.19$ \\
\hline $3364.9763 \ldots \ldots \ldots \ldots \ldots \ldots$ & $-41.36 \pm 3.23$ \\
\hline 3365.8259. & $-40.73 \pm 2.89$ \\
\hline $3367.8213 \ldots$ & $-64.14 \pm 2.93$ \\
\hline $3383.9213 \ldots$ & $-64.01 \pm 3.37$ \\
\hline $3390.7526 \ldots .$. & $-27.69 \pm 2.82$ \\
\hline $3448.7387 \ldots$ & $33.62 \pm 1.59$ \\
\hline $3451.7306 \ldots$ & $27.48 \pm 2.41$ \\
\hline $3476.6500 \ldots$ & $-118.87 \pm 2.57$ \\
\hline $3480.6372 \ldots \ldots$ & $-127.55 \pm 5.68$ \\
\hline 3481.6318..................... & $-82.68 \pm 2.08$ \\
\hline 3482.6317. & $-57.03 \pm 2.13$ \\
\hline $3664.9959 \ldots$ & $141.47 \pm 1.96$ \\
\hline $3675.9704 .$. & $127.44 \pm 2.24$ \\
\hline $3676.9844 \ldots \ldots$ & $123.13 \pm 3.69$ \\
\hline $3682.9515 \ldots \ldots \ldots \ldots \ldots \ldots$ & $20.69 \pm 2.78$ \\
\hline 3687.9321.................... & $15.18 \pm 2.50$ \\
\hline $3689.9285 \ldots \ldots$ & $61.28 \pm 2.54$ \\
\hline $3691.9156 \ldots$. & $92.73 \pm 2.98$ \\
\hline $3697.9126 \ldots \ldots \ldots \ldots \ldots \ldots$ & $123.87 \pm 2.03$ \\
\hline 3703.8858. & $147.52 \pm 4.60$ \\
\hline $3708.8817 \ldots$ & $146.26 \pm 3.24$ \\
\hline $3710.8789 \ldots$ & $150.70 \pm 2.58$ \\
\hline $3718.0141 \ldots$. & $136.36 \pm 4.98$ \\
\hline $3724.8303 \ldots \ldots$ & $129.03 \pm 3.12$ \\
\hline $3728.8252 \ldots$ & $121.82 \pm 2.94$ \\
\hline $3731.9672 \ldots \ldots$ & $83.91 \pm 3.86$ \\
\hline $3733.8020 \ldots \ldots$ & $39.79 \pm 3.20$ \\
\hline $3734.8087 \ldots \ldots \ldots \ldots \ldots \ldots . . . . . . .$. & $-3.84 \pm 2.64$ \\
\hline $3736.9455 \ldots$ & $-86.32 \pm 3.54$ \\
\hline $3741.7833 \ldots$ & $60.98 \pm 2.69$ \\
\hline $3742.7830 \ldots$ & $73.14 \pm 2.33$ \\
\hline $3743.7904 \ldots \ldots \ldots \ldots \ldots \ldots$ & $83.45 \pm 2.72$ \\
\hline $3748.7726 \ldots \ldots \ldots \ldots \ldots \ldots$ & $128.14 \pm 3.04$ \\
\hline $3751.7677 \ldots \ldots \ldots \ldots \ldots \ldots \ldots$ & $139.71 \pm 3.00$ \\
\hline $3753.7634 \ldots \ldots \ldots \ldots \ldots \ldots$ & $150.89 \pm 2.61$ \\
\hline $3754.7496 \ldots \ldots \ldots \ldots \ldots \ldots \ldots$ & $141.44 \pm 2.43$ \\
\hline $3756.7485 \ldots \ldots \ldots \ldots \ldots \ldots$ & $141.78 \pm 3.11$ \\
\hline $3764.7353 \ldots \ldots$ & $140.77 \pm 2.69$ \\
\hline $3832.6754 \ldots$ & $100.92 \pm 2.17$ \\
\hline $3833.6962 .$. & $94.87 \pm 2.55$ \\
\hline $3834.6755 \ldots$ & $85.38 \pm 1.92$ \\
\hline $3835.6671 \ldots \ldots$ & $63.55 \pm 2.01$ \\
\hline $3838.6638 \ldots \ldots \ldots \ldots \ldots \ldots \ldots$ & $-35.39 \pm 1.96$ \\
\hline $3841.6443 \ldots \ldots \ldots \ldots \ldots \ldots \ldots$ & $-46.03 \pm 4.92$ \\
\hline $3845.6318 \ldots \ldots \ldots$ & $72.93 \pm 2.72$ \\
\hline $3846.6533 \ldots \ldots \ldots \ldots \ldots \ldots$ & $77.92 \pm 2.69$ \\
\hline 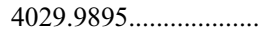 & $134.42 \pm 2.58$ \\
\hline $4035.9910 \ldots \ldots \ldots \ldots \ldots \ldots . . . . . . . .$. & $116.77 \pm 3.07$ \\
\hline 4038.9782. & $102.21 \pm 1.65$ \\
\hline 4039.9707.................. & $102.13 \pm 2.46$ \\
\hline 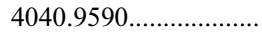 & $79.20 \pm 2.02$ \\
\hline $4043.9637 \ldots \ldots \ldots \ldots \ldots \ldots \ldots$ & $11.48 \pm 1.25$ \\
\hline $4044.9538 \ldots \ldots \ldots \ldots \ldots \ldots \ldots$ & $-18.63 \pm 1.81$ \\
\hline $4045.9556 \ldots \ldots \ldots \ldots \ldots \ldots . . . . . .$. & $-72.29 \pm 2.05$ \\
\hline $4050.9539 \ldots \ldots \ldots \ldots \ldots \ldots$ & $42.33 \pm 2.30$ \\
\hline 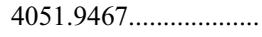 & $52.82 \pm 2.67$ \\
\hline $4052.9393 \ldots \ldots \ldots \ldots \ldots . . . . . . .$. & $67.80 \pm 1.72$ \\
\hline $4073.8806 \ldots$ & $125.65 \pm 1.92$ \\
\hline
\end{tabular}

TABLE 1-Continued

\begin{tabular}{|c|c|}
\hline HJD $-2,450,000.0$ & $\begin{array}{c}\mathrm{RV} \\
\left(\mathrm{m} \mathrm{s}^{-1}\right)\end{array}$ \\
\hline $4079.8634 \ldots \ldots$ & $130.08 \pm 2.64$ \\
\hline $4087.8437 \ldots \ldots$ & $95.94 \pm 1.78$ \\
\hline $4106.7835 \ldots \ldots \ldots \ldots \ldots \ldots .$. & $63.79 \pm 2.55$ \\
\hline 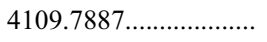 & $93.54 \pm 2.53$ \\
\hline $4110.7994 \ldots \ldots$ & $106.19 \pm 2.29$ \\
\hline $4129.8706 \ldots \ldots \ldots \ldots \ldots \ldots$ & $105.98 \pm 2.14$ \\
\hline $4130.7409 \ldots \ldots \ldots \ldots \ldots \ldots$ & $103.57 \pm 2.36$ \\
\hline $4133.8470 \ldots \ldots \ldots \ldots \ldots \ldots$ & $88.55 \pm 2.69$ \\
\hline $4134.7264 \ldots \ldots \ldots \ldots \ldots \ldots . .$. & $92.38 \pm 2.58$ \\
\hline $4135.8673 \ldots \ldots \ldots \ldots \ldots \ldots . .$. & $103.20 \pm 2.85$ \\
\hline $4136.8409 \ldots \ldots \ldots \ldots \ldots \ldots . .$. & $90.93 \pm 3.26$ \\
\hline $4148.6771 \ldots \ldots \ldots \ldots \ldots \ldots . .$. & $-81.12 \pm 3.41$ \\
\hline $4156.6604 \ldots \ldots \ldots \ldots \ldots \ldots . .$. & $48.73 \pm 3.71$ \\
\hline $4159.7804 \ldots \ldots \ldots \ldots \ldots \ldots \ldots . .$. & $66.51 \pm 2.32$ \\
\hline $4166.7627 \ldots \ldots \ldots \ldots \ldots \ldots .$. & $89.64 \pm 2.10$ \\
\hline $4167.7585 \ldots \ldots$ & $85.27 \pm 2.00$ \\
\hline $4211.6299 \ldots \ldots$ & $67.62 \pm 2.47$ \\
\hline $4231.6001 \ldots \ldots \ldots \ldots \ldots \ldots . .$. & $104.84 \pm 1.78$ \\
\hline
\end{tabular}

in the discovery paper $\left(M_{A}=1.27 M_{\odot}\right)$, which was estimated by Santos et al. (2003). We elected to adopt the former value in this paper for consistency with our previous work. We included the uncertainty suggested by Takeda et al. (2007) in the calculation of the planet minimum masses given in $\S 4$.

\section{RADIAL VELOCITY MEASUREMENTS}

We made high-precision radial velocity measurements of HD 74156 using the iodine absorption cell method (e.g., Butler et al. 1996). The specific details of our implementation are the same as for our previous work described in Bean et al. (2007).

Observations using the HET to feed the High Resolution Spectrograph (HRS; Tull 1998) were carried out by on-site telescope operators during 82 nights between UT dates 2004 December 3 and 2007 May 11. The HRS was used in the resolution $R=$ 60,000 mode with a 316 line $\mathrm{mm}^{-1}$ eschelle grating. The crossdispersion grating was positioned so that the central wavelength of the order that fell in the break between the two CCD chips was $5936 \AA$. A temperature-controlled cell containing molecular iodine gas $\left(\mathrm{I}_{2}\right)$ was inserted in front of the spectrograph slit entrance during all exposures to imprint lines that provided a contemporaneous wavelength scale and instrumental profile fiducial for the highprecision radial velocity measurements. The exposure times were nominally $150 \mathrm{~s}$, but varied up to 3 times that occasionally to account for increased seeing and/or cloud cover. Three sequential exposures were obtained each night and a total of 242 spectra of HD 74156 for radial velocity measurements were collected.

HD 74156 was also observed once on 2006 December 14 without the iodine cell and with the same instrument setup, but in the $R=120,000$ mode. The exposure time was $650 \mathrm{~s}$. The spectrum from this observation served as a template for the radial velocity measurements.

CCD reduction and optimal spectral extraction were carried out for all the individual spectra using the REDUCE package (Piskunov \& Valenti 2002) and nightly calibration data. Relative radial velocities were measured from the spectra using the modeling algorithm that is described in detail by Bean et al. (2007). We used the GaussFit program (Jefferys et al. 1988) to reduce each of the 82 multiple observation sets to a single velocity. The velocities we measured are relative to an arbitrary zero point, and 
TABLE 2

The Radial Velocity Samples

\begin{tabular}{|c|c|c|c|}
\hline Sample & Time Span & $N$ & $\begin{array}{c}\mathrm{rms} \\
\left(\mathrm{m} \mathrm{s}^{-1}\right)^{\mathrm{a}}\end{array}$ \\
\hline ELODIE ....................... & $1998.02-2003.37$ & 48 & 9.5 \\
\hline CORALIE ..................... & $2001.03-2003.74$ & 38 & 11.1 \\
\hline 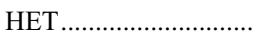 & $2004.92-2007.36$ & 82 & 6.0 \\
\hline
\end{tabular}

a The rms of the residuals from the Robust three-planet model.

we determined an offset simultaneously with our orbit analysis to adjust them to the system barycenter (see $\S 4$ ). The final velocities with this offset subtracted are given in Table 1 . They have a median uncertainty of $2.6 \mathrm{~m} \mathrm{~s}^{-1}$.

\section{ORBIT ANALYSIS}

We combined our measured radial velocities for HD 74156 with those from Naef et al. (2004; the "ELODIE" and "CORALIE" samples) to create a data set that spans $9.33 \mathrm{yr}$. No other highprecision radial velocities for HD 74156 have been published. The timespan, number of data points, and rms residuals to the final best-fit model for each of the three individual velocity samples are given in Table 2. The ELODIE and CORALIE measurements overlap, but there is a gap of $1.19 \mathrm{yr}$ between the final CORALIE measurement and our first measurement with the HET.

We fit the total radial velocity data set with a double-Keplerian model to determine the two previously known planets' period $P$, velocity semiamplitude $K$, eccentricity $e$, longitude of periastron $\omega$, and time of periastron $T_{P}$. To account for the heterogeneous nature of the data set, we also simultaneously determined offset parameters for each individual velocity sample to adjust them to be relative to the system barycenter. We performed the fitting using the GaussFit program (Jefferys et al. 1988) with both robust and least-squares ("reduced chi-squared" metric, $\chi_{\nu}^{2}$ ) estimation to determine the parameter values that gave the best match between our model and the measured data. For our robust fitting we used the "fair" metric described by Rey (1983) with an adopted asymptotic relative efficiency (ARE) of 0.92 . We refer to the models found with the two methods as "Robust" and "Least Squares" hereafter. In the case of the two-planet model, the parameters determined using the two methods were consistent.

To search for evidence of additional planets in the HD 74156 system we calculated a periodogram (Press et al. 1992) of the velocity residuals from the two-planet model fit. The result is shown in Figure 1. The highest peak in the periodogram is at a period around 349 days and has a formal false alarm probability (FAP) of $0.0014 \%$. We used the bootstrap method described by Endl et al. (2002) to further investigate the likelihood that this signal was real. We calculated a periodogram and noted the maximum power for each of 10,000 simulated data sets. The data sets were generated by randomly scrambling the two-planet model residuals while maintaining the sampling of the observations. We did not find a maximum power in any of the trial periodograms as high as or higher than the peak at 349 days in the periodogram of the real two-planet model residuals (19.98). This implies a FAP for the 349 day signal of less than $0.01 \%$, which is consistent with the formal estimate. We took this as evidence for a real periodic signal in the radial velocities not accounted for by the two previously known planets.

We explored the possibility that the detected periodic signal is attributable to a third planet in the system by fitting a tripleKeplerian model to the radial velocity data set. We again used both the robust and least-squares estimation methods. The quality

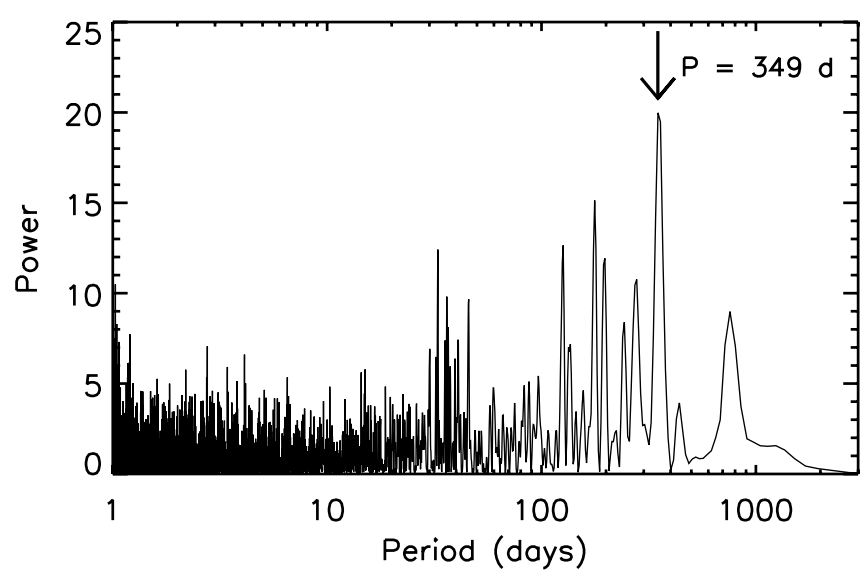

FIG. 1.-Periodogram of the velocity residuals from the two-planet Robust model. The spike around 349 days indicates a remaining periodic signal that is not accounted for by the previously known planets. The false alarm probability of the peak is $0.0014 \%$.

of the fits improved significantly with the addition of the third component. However, we found that the rms of the HET velocity residuals $\left(6.0 \mathrm{~m} \mathrm{~s}^{-1}\right)$ remained more than twice the median of the measurement uncertainties $\left(2.6 \mathrm{~m} \mathrm{~s}^{-1}\right)$. This discrepancy could be due to using an incomplete model of the system (i.e., there are more than three planets), intrinsic variations of the star's photosphere that mimic real radial velocity changes, improper weighting of the ELODIE and CORALIE data, and/or errors in our velocity measurement method. Using the method of Wright (2005), Butler et al. (2006) estimated the radial velocity "jitter" for HD 74156 to be $4.0 \mathrm{~m} \mathrm{~s}^{-1}$. Adding this value in quadrature with the HET velocity uncertainties yields errors that are slightly above what would be expected from the fit residuals and the fit $\chi_{\nu}^{2}=$ 0.88 . This is not surprising, because our method of taking multiple measurements over 10-25 minutes and combining them into one measurement reduces the noise arising from short-term stellar variability. We ultimately decided to increase the HET uncertainties by a factor of 2 in order to account for potential errors in the data and to avoid overweighting them in the orbital fitting, but we cannot discount the possibility that there are additional planets in the system causing the higher than expected dispersion. Therefore, we have elected to publish our original estimate of the HET velocity uncertainties in Table 1 to avoid biasing future investigations.

We repeated both the two- and three-planet orbit fitting using the revised uncertainties for the HET data. The orbital parameters and associated uncertainties that we determined for the threeplanet models and minimum masses calculated from these parameters are given in Table 3 . We found that the $\chi_{\nu}^{2}$ of the Least Squares model improved from 2.3 to 1.4 by introducing the third planet into the model. The rms of the HET velocity residuals from the fits improved from 8.5 to $6.0 \mathrm{~m} \mathrm{~s}^{-1}$ for both methods. The rms for the ELODIE residuals went from 12.7 to $10.8 \mathrm{~m} \mathrm{~s}^{-1}$ in the Least Squares model and from 12.8 to $9.5 \mathrm{~m} \mathrm{~s}^{-1}$ in the Robust model. However, the rms of the CORALIE residuals degraded from 10.3 to $10.5 \mathrm{~m} \mathrm{~s}^{-1}$ in the Least Squares model and from 10.2 to $11.1 \mathrm{~m} \mathrm{~s}^{-1}$ in the Robust model. The three-planet model does yield an improved fit to the combined ELODIE and CORALIE data set, with the rms of the residuals from the Robust model improving from 11.7 to $10.3 \mathrm{~m} \mathrm{~s}^{-1}$.

The HET velocities and the Robust fit are shown in Figure 2. The radial velocity data are shown phased to each component's period and with the other two components' orbits subtracted assuming the Robust model in Figure 3. The Robust and Least 
TABLE 3

Derived Parameters for the HD 74156 Planets

\begin{tabular}{|c|c|c|c|}
\hline Parameter & HD 74156 b & HD $74156 \mathrm{c}$ & HD $74156 \mathrm{~d}$ \\
\hline \multicolumn{4}{|c|}{ Robust Solution } \\
\hline$P$ (days) & $51.65 \pm 0.01$ & $2476.7 \pm 8.7$ & $346.6 \pm 3.6$ \\
\hline$K\left(\mathrm{~m} \mathrm{~s}^{-1}\right)$ & $115.1 \pm 1.5$ & $115.5 \pm 2.3$ & $10.5 \pm 1.2$ \\
\hline$T_{0}(\mathrm{HJD}-2,450,000.0) \ldots .$. & $1980.8 \pm 0.1$ & $952.2 \pm 13.8$ & $678.2 \pm 44.2$ \\
\hline$e_{\text {end }}$ & $0.64 \pm 0.01$ & $0.43 \pm 0.01$ & $0.25 \pm 0.11$ \\
\hline$\omega(\operatorname{deg})$ & $175.8 \pm 0.8$ & $261.3 \pm 2.0$ & $166.5 \pm 27.4$ \\
\hline$M \sin i\left(M_{\mathrm{Jup}}\right)^{\mathrm{a}} \ldots \ldots \ldots \ldots \ldots \ldots$ & $1.88 \pm 0.03$ & $8.03 \pm 0.12$ & $0.40 \pm 0.02$ \\
\hline \multicolumn{4}{|c|}{ Least-Squares Solution } \\
\hline$P$ (days).. & $51.65 \pm 0.01$ & $2481.8 \pm 8.5$ & $339.6 \pm 2.6$ \\
\hline$K\left(\mathrm{~m} \mathrm{~s}^{-1}\right) \ldots \ldots$ & $114.9 \pm 1.8$ & $118.1 \pm 2.8$ & $13.5 \pm 2.0$ \\
\hline$T_{0}(\mathrm{HJD}-2,450,000.0) \ldots \ldots$ & $1980.8 \pm 0.1$ & $927.6 \pm 14.3$ & $757.5 \pm 25.7$ \\
\hline 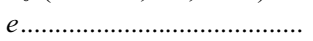 & $0.64 \pm 0.01$ & $0.44 \pm 0.01$ & $0.55 \pm 0.07$ \\
\hline$\omega(\operatorname{deg})$ & $175.8 \pm 0.8$ & $257.6 \pm 2.3$ & $188.1 \pm 11.1$ \\
\hline$M \sin i\left(M_{\text {Jup }}\right)^{\mathrm{a}} \ldots \ldots \ldots \ldots \ldots \ldots$ & $1.87 \pm 0.03$ & $8.19 \pm 0.13$ & $0.45 \pm 0.04$ \\
\hline
\end{tabular}

${ }^{a}$ Assuming $M_{\star}=1.24 \pm 0.04 M_{\odot}$ (Takeda et al. 2007)

Squares models for the two previously known planets are very similar. However, the period, time of periastron, and eccentricity for the candidate third planet determined using the two different methods are inconsistent. The most notable difference between the models for this planet is in the derived eccentricities, with the Robust model having $e=0.25$ and the Least Squares model having $e=0.55$. For comparison, the new planet's phased orbit for the Least Squares model is shown in Figure 4.

The least-squares method of fitting involves weighting the data points according to their input variances and is based on the assumption that the data are normally distributed. The purpose of using robust estimation instead is to reduce the influence of nonnormal outliers in the data. Rather than attempting to identify outliers by hand, robust metrics place outlier identification and their subsequent reweighting on a rigorous footing. Data points with fit residuals larger than a certain threshold are iteratively down-weighted, rather than completely ignored, during the fitting process. However, the identification of some data points as outliers does not mean they are necessarily bad. It only means that they do not agree with adopted physical and noise models and thus should not carry their normal weight when assessing the fit quality to avoid biasing the final solution. For example, a small signal due to a fourth planet in the system could cause some data points to seem highly discrepant. In this case the data points would be correct, but would adversely affect the fitting of a model that only includes three components if they were not identified and down-weighted.

The similarity of the derived orbital parameters for the two previously known planets when using the robust and least-squares methods is due to the fact that even the worst possible outliers in the radial velocity data are small relative to the amplitudes of the planets' velocity signals. However, the size of the outliers is the same or even larger than the amplitude of the velocity signal from the possible third planet. Therefore, whether potential outliers are identified and treated differently significantly influences the orbital solution for this component.

The Robust model for HD $74156 \mathrm{~d}$ is based on the estimation that some of the HET data points are actually contaminating outliers. This is not surprising given the variety of physical processes that can influence a measured stellar radial velocity at the $2-3 \mathrm{~m} \mathrm{~s}^{-1}$ level. The down-weighting of the identified outliers leads to a less eccentric orbit as the fit to highly deviant points with small input uncertainties is relaxed. The Least Squares model is the best fit to the data assuming that the input errors are scaled correctly and the data are normally distributed. The weight given to all the HET data points is based on their input uncertainties, which are twice those given in Table 1. The result is a more eccentric orbit for the new planet because the solution is strongly influenced by the potential outliers. We favor the Robust model over the Least Squares model, but we chose to present and discuss both in order to illustrate the uncertainty in derived orbital parameters for planets when the signal is near the detection threshold in the data.

A periodogram of the three-planet Robust model residuals is shown in Figure 5. The periodogram of the residuals to the Least Squares model is essentially the same. The addition of the third component to the model removes the periodicity detected at 349 days. No periodicity is detected in the three-planet model residuals with a FAP less than $22 \%$. This indicates that a summation of three Keplerian orbits adequately accounts for the detected periodic signals in the radial velocities.

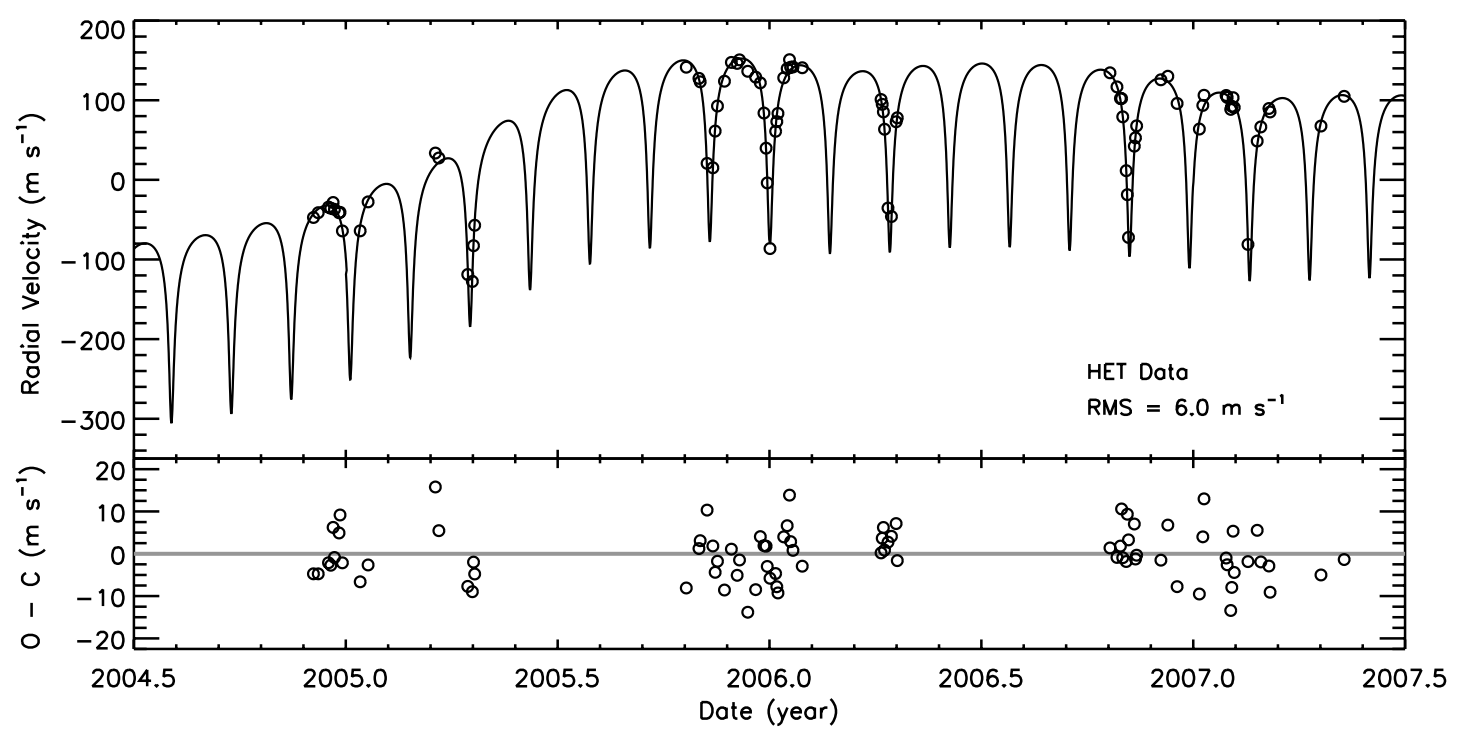

FIG. 2.-Top: HET radial velocities (circles) and the Robust three-planet model to all the data (line). Bottom: Residuals from the fit (circles). The error bars are omitted for clarity. 

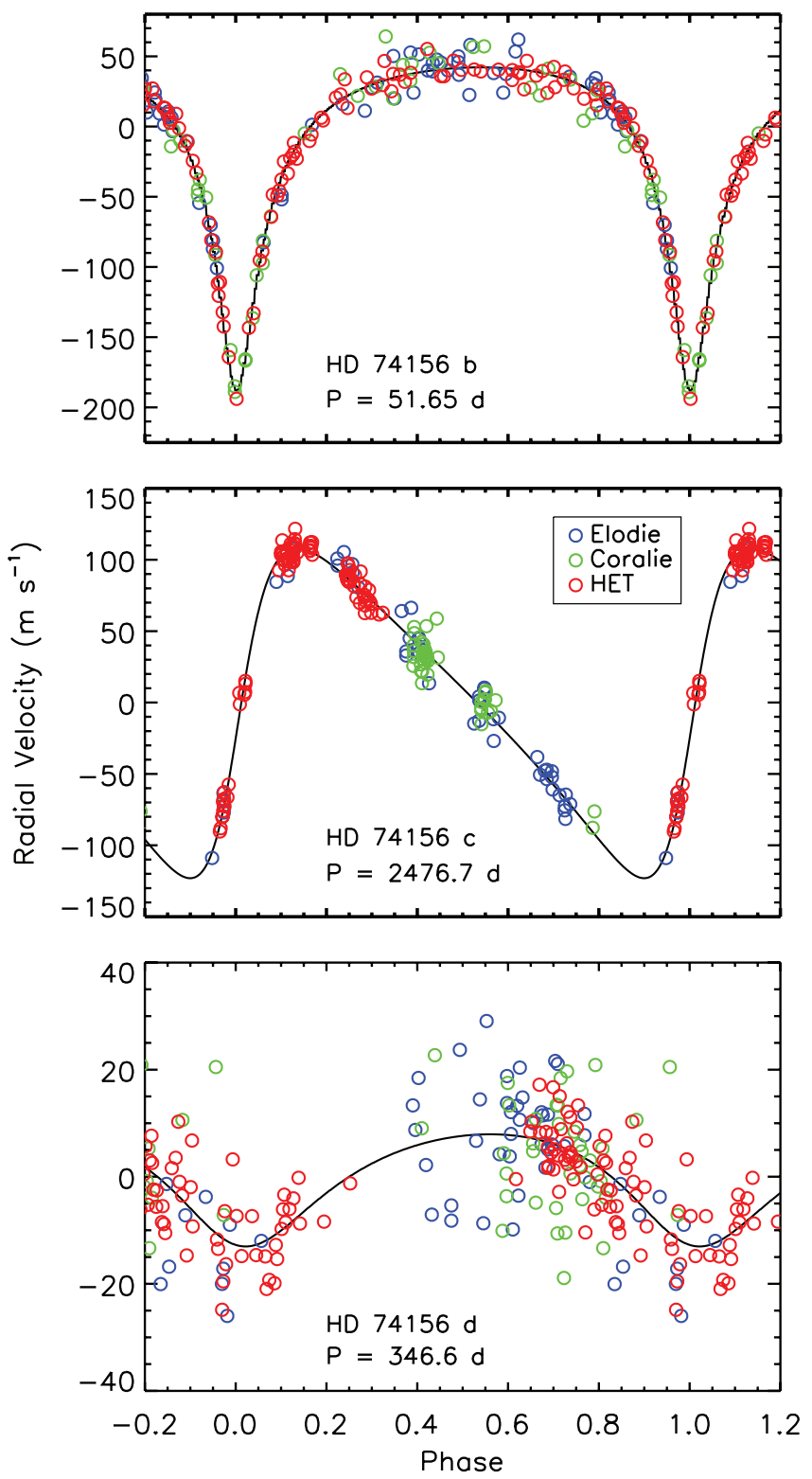

FIG. 3. - Representation of the Robust three-planet model. The radial velocity data (circles) are phased to each component's period, with the other two components' orbits subtracted, and the component's model velocities (lines). The error bars are omitted for clarity.

We note that the periodogram of the two-planet model residuals in Figure 1 also exhibits moderately significant power around 175 days. This is roughly half the value of the period we explored and ultimately determined for the potential third planet. Also, the 175 day spike in the two-planet model residual periodogram has a smaller power ( $\mathrm{FAP}=0.09 \%$ from the bootstrap simulation) than the spike at 349 days and is not present in the periodogram of the three-planet model residuals. We therefore suspect that the smaller, moderately significant spikes around 175 days in the periodogram of the two-planet model residuals were aliases. Nevertheless, we attempted to fit a three-planet model to the radial velocity data where the third component had a period around 175 days. We were not able to determine a physically realistic set of parameters for the third component with this approach, and various trials with fixed parameters yielded much worse fits than the three-planet fits where the third component had a period close to 349 days $\left(\Delta \chi^{2}>80\right.$ for the least-squares approach).

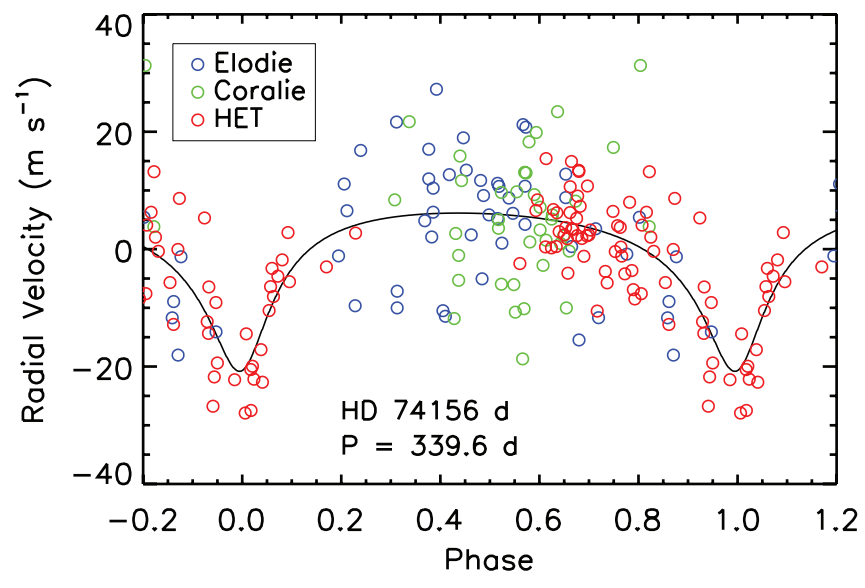

FIG. 4.-Representation of the Least Squares orbit for the third planet (line) with the other two components' orbits subtracted from the data (circles). Note that the small differences in the parameters for the two other planets do yield slightly different residuals when their orbits are subtracted from the data. The error bars are omitted for clarity.

The significant improvement in fit quality for both the Robust and Least Squares models and comparison of the two-planet and three-planet model residual periodograms lead us to propose that there is a third planet in the HD 74156 system with orbital parameters similar to those given in Table 3. Beyond the detection of this new planet, our data and analysis have also yielded improved orbital parameters for HD $74156 \mathrm{c}$. We find for this planet a period $22 \%$ longer and an eccentricity reduced by 0.13 from the values determined by Naef et al. (2004). This is not an unusual result when determining revised orbital parameters for long-period planets with the addition of significantly more radial velocity data.

From our determined Robust model parameters we estimate $M_{b} \sin i=1.88 \pm 0.03, M_{c} \sin i=8.03 \pm 0.12$, and $M_{d} \sin i=$ $0.40 \pm 0.02 M_{\text {Jup }}$ assuming $M_{A}=1.24 \pm 0.04 M_{\odot}$ (Takeda et al. 2007). The derived velocity semiamplitude for the new planet $\left(\sim 11.5 \mathrm{~m} \mathrm{~s}^{-1}\right)$ is roughly the same magnitude as the ELODIE and CORALIE residuals, while it is roughly twice that of the HET residuals. Therefore, the HET velocities are most sensitive to the third planet. Its period is also close to $1 \mathrm{yr}$ (but significantly different enough so that it cannot be due an alias or error in the data) and the $2.5 \mathrm{yr}$ of HET observations do not give full phase coverage. More high-precision observations over the next few years will be needed to refine the orbital parameters for this planet.

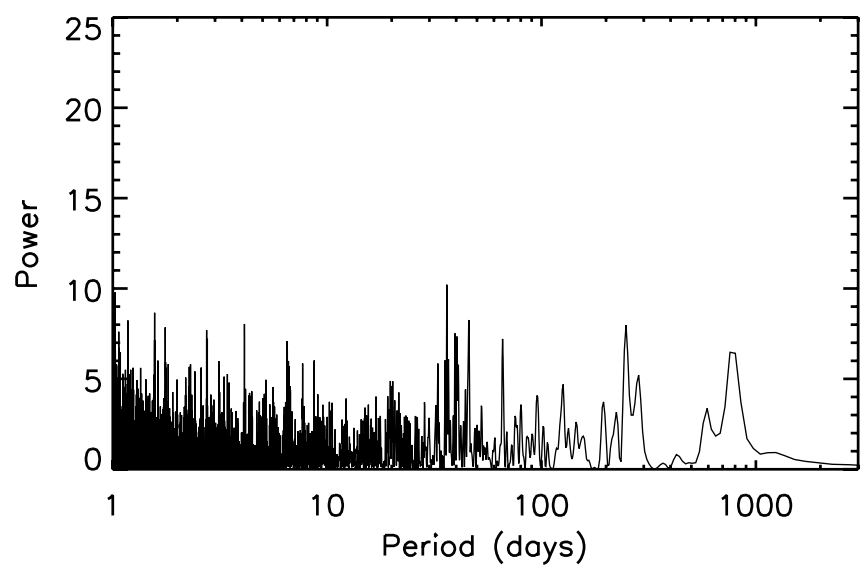

FIG. 5.-Periodogram of the residuals to the three-planet Robust model. No periodicity is detected with a false alarm probability less than $22 \%$. 
TABLE 4

Orbital Parameters for Planets in Systems with Three or More Planets

\begin{tabular}{|c|c|c|c|}
\hline Planet & $\begin{array}{c}P \\
\text { (days) }\end{array}$ & $e$ & Source \\
\hline 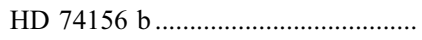 & 51.65 & 0.64 & 1 \\
\hline 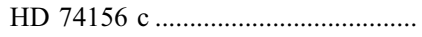 & 2476.7 & 0.43 & 1 \\
\hline 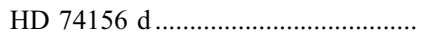 & 346.6 & 0.25 & 1 \\
\hline GJ 876 b ........... & 60.83 & 0.03 & 2 \\
\hline GJ 876 c.......... & 30.46 & 0.26 & 2 \\
\hline GJ $876 \mathrm{~d} \ldots \ldots .$. & 1.94 & 0.00 & 2 \\
\hline$\rho^{1} \mathrm{Cnc} \mathrm{b} . . . . .$. & 14.65 & 0.01 & 3 \\
\hline$\rho^{1} \mathrm{Cnc} \mathrm{c} \ldots \ldots \ldots$ & 44.36 & 0.07 & 3 \\
\hline$\rho^{1} \mathrm{Cnc} \mathrm{d} \ldots \ldots . . .$. & 5552 & 0.09 & 3 \\
\hline 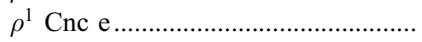 & 2.80 & 0.09 & 3 \\
\hline$v$ And $\mathrm{b} \ldots \ldots \ldots . .$. & 4.62 & 0.02 & 3 \\
\hline 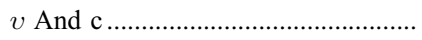 & 241.2 & 0.26 & 3 \\
\hline$v$ And $\mathrm{d} \ldots \ldots \ldots . . .$. & 1290.1 & 0.26 & 3 \\
\hline 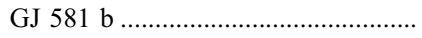 & 5.37 & 0.02 & 4 \\
\hline 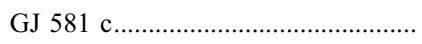 & 12.93 & 0.16 & 4 \\
\hline 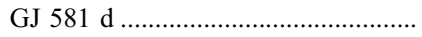 & 83.6 & 0.20 & 4 \\
\hline HD $69830 \mathrm{~b} \ldots$ & 8.67 & 0.10 & 5 \\
\hline HD $69830 \mathrm{c} \ldots \ldots .$. & 31.56 & 0.13 & 5 \\
\hline HD $69830 \mathrm{~d} \ldots$. & 197.0 & 0.07 & 5 \\
\hline 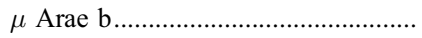 & 643.25 & 0.13 & 6 \\
\hline 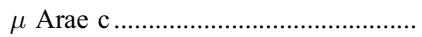 & 9.64 & 0.17 & 6 \\
\hline$\mu$ Arae d........... & 310.55 & 0.07 & 6 \\
\hline$\mu$ Arae e & 4205.8 & 0.10 & 6 \\
\hline 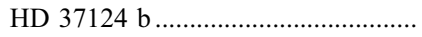 & 154.46 & 0.06 & 3 \\
\hline 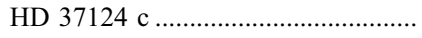 & 2295 & 0.20 & 3 \\
\hline 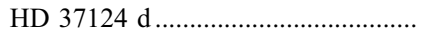 & 843.6 & 0.14 & 3 \\
\hline
\end{tabular}

RefERENCES.-(1) This paper; (2) Rivera et al. 2005; (3) Butler et al. 2006; (4) Udry et al. 2007; (5) Lovis et al. 2006; (6) Pepe et al. 2007.

The eccentricities we have determined by fitting the radial velocity data indicate that the two previously known planets are in highly eccentric orbits, while the new planet is in at least a moderately eccentric orbit. This raises the question of whether the derived orbital parameters represent a stable three planet system. We made a preliminary check of this using the Runge Kutta numerical integrator in the Systemic Console. ${ }^{5}$ We integrated the planet positions forward in time for $1000 \mathrm{yr}$ assuming their true masses were equal to the minimum masses and that their orbits were in the same plane. We used a 0.05 day time step for the calculations. The $1000 \mathrm{yr}$ integration represents about 7100 full orbits of the innermost planet. We found that the semimajor axes of all three planets were not predicted to change from their initial values during the entirety of both integrations. Therefore, both sets of orbital parameters that we have determined do not describe highly unstable systems.

Prior to our discovery, Raymond \& Barnes (2005) had calculated that an additional Saturn-mass planet in the HD 74156 system having an orbital semimajor axis $a=0.9-1.4 \mathrm{AU}$, and $e<$ 0.15 would very likely be dynamically stable for 100 million yr. They had even predicted that such a planet exists based on their "Packed Planetary Systems" hypothesis (Barnes \& Raymond 2004). The candidate planet we have identified has $M \sin i=$ $1.3 M_{\text {Sat }}$ and $a=1.0 \mathrm{AU}$, which is very similar to the properties of their stable test planet. However, the simulations presented by Raymond \& Barnes (2005) were based on orbital parameters for the previously known planets that are somewhat different than we have determined based on the new radial velocity data, and

\footnotetext{
5 Available at http://www.oklo.org. We used the 2007 May 30 version of the code.
}

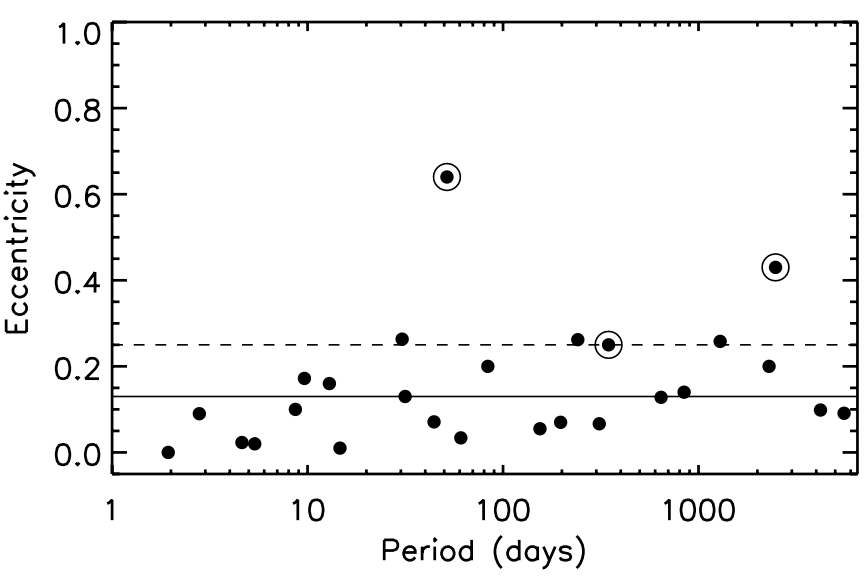

FIG. 6.-Orbital eccentricity as a function of period for the planets in the eight systems containing three or more planets ( filled circles; see Table 4 for the data and sources). The median value for this sample is 0.13 (solid line), while the median orbital eccentricity of all the known exoplanets is 0.25 (dashed line). The planets in the HD 74156 system (open circles) are notably eccentric relative to the planets in the systems with three or more planets. Note that the orbital eccentricity of $\operatorname{HD} 74156 \mathrm{~d}(P=347$ days $)$ is poorly constrained with the current data.

we find a slightly higher eccentricity for the new third planet than they considered. Nevertheless, the results of their robust simulations support our proposal that the third planet exists and that the orbital parameters we have derived for it are plausible.

\section{DISCUSSION}

With our detection of a third planet around HD 74156, it becomes the eighth normal star to host three or more planets. Currently, five other stars are known to host three planets (GJ 876, $v$ And, GJ 581, HD 69830, and HD 37124), and two are known to host four planets ( $\rho^{1} \mathrm{Cnc}$ and $\mu$ Arae). The most recently determined orbital eccentricities and periods for these planets are tabulated along with the source of the data in Table 4. These same data are plotted in Figure 6. The planets in systems containing three or more planets have a median orbital eccentricity of 0.13 , which is significantly lower than the median of 0.25 for all known exoplanets. This could be due to the increased likelihood of dynamical instabilities developing in higher order planetary systems where one or more component has a significantly elliptical orbit. Indeed, Chatterjee et al. (2007) have proposed that dynamical instabilities leading to planet ejection can quickly arise in systems initially containing three gas giants without requiring special orbital configurations or additional evolutionary mechanisms. It might then be expected that the three-planet systems which survive for the timescale of the typical known exoplanet host star's age (i.e., those that are detectable with current techniques) would be those with planets having low orbital eccentricities.

This hypothesis seems to be supported by the sample of previously known systems containing three or more planets. However, HD $74156 \mathrm{~b}$ and $\mathrm{c}$ are in orbits that are much more eccentric than any of the components in these systems. Is HD 74156 a rare example of a system that survived a period of dynamical instability with three gas giants in highly eccentric orbits, or does its existence point to other mechanisms as playing an important role in the evolution of planetary systems? Studies of the dynamical characteristics of HD 74156 under the assumption of the previous two-planet model have been carried out by Nagasawa et al. (2003), Barnes \& Raymond (2004), Raymond \& Barnes (2005), Raymond et al. (2006), Adams \& Laughlin (2006), Barnes \& Greenberg (2006), and Libert \& Henrard (2006, 2007). Clearly, new dynamical 
studies should be undertaken now that we have uncovered the third planet in order to understand the possible origins of this system's unique configuration.

We would like to thank Eugenio Rivera, Stefano Meschiari, Aaron Wolf, and Greg Laughlin for providing the Systemic Console to the community. We also thank the anonymous referee for a careful reading of the manuscript and constructive comments which improved this paper. We acknowledge support from NASA GO-09407, GO-09969, GO-09971, GO-10103, GO-10610, and GO-10989 from the Space Telescope Science Institute, which is operated by the Association of Universities for Research in Astronomy, Inc., under NASA contract NAS5-26555; and from JPL 1227563 (SIM MASSIF Key Project: P.I. Todd Henry), administered by the Jet Propulsion Laboratory.
Adams, F., \& Laughlin, G. 2006, ApJ, 649, 992

Barnes, R., \& Greenberg, R. 2006, ApJ, 652, L53

Barnes, R., \& Raymond, S. N. 2004, ApJ, 617, 569

Bean, J. L., McArthur, B. E., Benedict, G. F., Harrison, T. E., Bizyaev, D., Nelan, E., \& Smith, V. V. 2007, AJ, 134, 749

Benedict, G. F., et al. 2002, ApJ, 581, L115 2006, AJ, 132, 2206

Bouchy, F., et al. 2005, A\&A, 444, L15

Butler, R. P., Marcy, G. W., Williams, E., McCarthy, C., Dosanjh, P., \& Vogt, S. S. 1996, PASP, 108, 500

Butler, R. P., et al. 2006, ApJ, 646, 505

Castellano, T. 2000, PASP, 112, 821

Charboneau, D., Brown, T. M., Latham, D. W., \& Mayor, M. 2000, ApJ, 529, L45

Chatterjee, S., Ford, E. B., \& Rasio, F. A. 2007, ApJ, submitted (astro-ph/0703166)

Chiang, E., \& Murray, N. 2002, ApJ, 576, 473

Cowan, N. B., Agol, E., \& Charbonneau, D. 2007, MNRAS, 379, 641

Deming, D., Seager, S., Richardson, L. J., \& Harrington, J. 2005, Nature, 434, 740

Endl, M., Kürster, M., Els, S., Hatzes, A. P., Cochran, W. D., Dennerl, K., \& Döbereiner, S. 2002, A\&A, 392, 671

ESA 1997, The Hipparcos and Tycho Catalogues (ESA SP-1200; Noordwijk: ESA)

Fischer, D. A., \& Valenti, J. 2005, ApJ, 622, 1102

Ford, E. B., Lystad, V., \& Rasio, F. A. 2005, Nature, 434, 873

Gillon, M., et al. 2007, A\&A, in press (arXiv: 0705.2219)

Harrington, J., et al. 2006, Science, 314, 623

Henry, G. W., Marcy, G. W., Butler, R. P., \& Vogt, S. S. 2000, ApJ, 529, L41

Janson, M., et al. 2007, AJ, 133, 2442

Jefferys, W. H., Fitzpatrick, M. J., \& McArthur, B. 1988, Celest. Mech., 41, 39

Knutson, H. A., et al. 2007, Nature, 447, 183

Laughlin, G., \& Adams, F. C. 1999, ApJ, 526, 881
REFERENCES

Libert, A.-S., \& Henrard, J. 2006, Icarus, 183, 186

2007, A\&A, 461, 759

Lòpez-Morales, M., Morrell, N. I., Butler, R. P., \& Seager, S. 2006, PASP, 118, 1506

Lovis, C., et al. 2006, Nature, 441, 305

McArthur, B. E., et al. 2004, ApJ, 614, L81

Naef, D., Mayor, M., Beuzit, J. L., Perrier, C., Queloz, D., Sivan, J. P., \& Udry, S. 2004, A\&A, 414, 351

Nagasawa, M., Lin, D. N. C., \& Ida, S. 2003, ApJ, 586, 1374

Pepe, F., et al. 2007, A\&A, 462, 769

Piskunov, N. E., \& Valenti, J. A. 2002, A\&A, 385, 1095

Press, W. H., Teukolsky, S. A., Vetterling, W. T., \& Flannery, B. P. 1992, Numerical Recipes: The Art of Scientific Computing (2nd ed.; Cambridge: Cambridge Univ. Press)

Raymond, S. N., \& Barnes, R. 2005, ApJ, 619, 549

Raymond, S. N., Barnes, R., \& Kaib, N. A. 2006, ApJ, 644, 1223

Rey, W. J. J. 1983, Introduction to Robust and Quasi-Robust Statistical Methods (New York: Springer)

Richardson, L. J., Deming, D., Horning, K., Seager, S., \& Harrington, J. 2007, Nature, 445, 892

Rivera, E. J., et al. 2005, ApJ, 634, 625

Santos, N. C., Israelian, G., Mayor, M., Rebolo, R., \& Udry, S. 2003, A\&A, 398,363

Sato, B., et al. 2005, ApJ, 633, 465

Shankland, P. D., et al. 2006, ApJ, 653, 700

Takeda, G., Ford, E. B., Sills, A., Rasio, F. A., Fischer, D. A., \& Valenti, J. A. 2007, ApJS, 168, 297

Tull, R. G. 1998, Proc. SPIE, 3355, 387

Udry, S., et al. 2007, A\&A, 469, L43

Valenti, J. A., \& Fischer, D. A. 2005, ApJS, 159, 141

Wright, J. T. 2005, PASP, 117, 657 